\title{
Global Analysis of a piecewise smooth epidemiological model of COVID-19
}

\author{
Tiago Carvalho $₫$ Rony Cristiano $₫$ \\ Diego S. Rodrigues $[$ - Durval J. Tonon $[$ ]
}

Received: 6 October 2020 / Accepted: 4 August 2021 / Published online: 24 August 2021

(C) The Author(s), under exclusive licence to Springer Nature B.V. 2021

\begin{abstract}
Despite the huge relevance of vaccines for preventing COVID-19, physical isolation and quarantine of infected individuals are still the key strategies to fight against the COVID-19 pandemic. Based on a COVID-19 transmission epidemiological model governed by ordinary differential equations, here we propose an intermittent non-pharmacological protocol to control the fraction of infected individuals. In our approach, unlike what generically happens for numerical simulation models, we provide a global analysis of the model, giving qualitative information about every initial condition. Under some simple hypothesis and variations of parameters, we present some bifurcations and we are able to predict the minimum social distancing effort that do not collapse the health system.
\end{abstract}

T. Carvalho $(\varangle)$

Department of Computing and Mathematics, Faculty of Philosophy, Sciences and Letters of Ribeirão Preto, Univ. of São Paulo, 14040-901 Ribeirão Preto, SP, Brazil e-mail: tiagocarvalho@usp.br

R. Cristiano · D. J. Tonon Institute of Mathematics and Statistics, Federal University of Goiás, Avenida Esperança s/n, Campus Samambaia, 74690-900 Goiânia, Goiás, Brazil

e-mail: rony.cristiano@ufg.br

D. J. Tonon

e-mail: djtonon@ufg.br

D. S. Rodrigues

School of Technology, University of Campinas, R. Paschoal Marmo, 1888, 13484-332 Limeira, SP, Brazil e-mail: diego.rodrigues@ft.unicamp.br
Keywords Piecewise smooth vector fields · Bifurcations · COVID-19 · Asymptotic stability

Mathematics Subject Classification 34A36 · 34C60

\section{Introduction}

Shortly after the raising of atypical pneumonia cases in Wuhan in the end of 2019, World Health Organization (WHO) has declared the novel coronavirus (COVID19) outbreak a global pandemic on March 11, 2020 [1]. Since then, many different public health actions have been taken as attempts to fight against the disease worldwide. For now, though, in spite of the huge efforts that have been made, no medication or drug can be considered as a reasonable solution capable of stopping or treating COVID-19.

Still in the pharmacological interventions, but in the field of prevention, it is unquestionable that vaccines certainly are great value and impact on avoiding new infections by SARS-CoV-2, and not only on the vaccinated people, but possibly on wider populations, due to the herd immunity effect [2]. However, the high levels of requests for vaccines and their limited production and distribution are still barriers to stopping COVID19 worldwide. Besides, it is not clear how long the immunization of a given vaccine can last. Meanwhile, non-pharmacological interventions like physical isolation and quarantine of infected individuals are the best 
current alternatives to locally control COVID-19 epidemic outbreaks.

Given the circumstances discussed in the preceding paragraphs, and since physical isolation and quarantine of infected individuals are still key strategies to fight against COVID-19, here we analyse the valuable SIR-X model setting developed by Maier and Brockmann [3] in the light of piecewise smooth dynamical systems theory. For the sake of completeness, one could say that the starting point of referred work is the classical SIR ordinary differential equation system, where the compartments $S, I$ and $R$ stands for susceptible, infected and removed individuals, ${ }^{1}$ respectively. In such a dynamics, the respective variables $S, I$ and $R$ usually denote the number of individuals in each one of these compartments as continuous-time functions of time $t$ [4].

The classical SIR ordinary differential equation system can be view as a particular case of the SIR-X dynamics given by Eqs. (1), (2), (3) and (4) with $\kappa=\kappa_{0}=0$ (i.e., with no isolation or quarantine). Given such SIR dynamics, the threshold that defines whether an epidemic will occur is the basic reproductive ratio $R_{0}=\alpha / \beta$. If both $R_{0}>1$ and the fraction of susceptibles is greater than $1 / R_{0}$, then there is an epidemic. This last condition generates a key threshold for vaccination strategies because vaccination transfers susceptibles to the removed compartment. Under the hypotheses of the SIR dynamics, but even though $R_{0}>1$, if the coverage of vaccination is greater than $1-\left(1 / R_{0}\right)$ in a certain population, then there is no sustained transmission in it $[2,4]$. Regarding COVID-19 epidemiological models, given social relevance on this subject, a large amount of peer-reviewed articles and preprints have been published, but sometimes with misuse of mathematical modeling [5]. The subject is quite wide and complex such that a review on this theme is beyond the scope of the paper [6,7]. Our key point here is to analyze, in the light of piecewise smooth dynamical systems theory, the new effective way proposed by Maier and Brockmann $[3,8]$ of modeling containment and quarantine.

In the relevant context of modeling the non-pharmacological interventions for COVID-19, the novelty proposed by Maier and Brockmann [3] regards two new

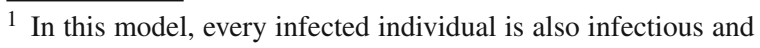
vice versa. Here in the paper, the compartments of removed individuals comprises both recovered and death individuals, i.e., the ones that do not take part in the disease transmission process.
}

processes: (1), general public containment efforts or individual behavioral changes in response to the epidemic effectively, which removes individuals from the interaction dynamics and (2), a quarantine procedure, in which symptomatic infected individuals are removed to quarantine. To model these processes, they introduce a new compartment for symptomatic, quarantined infecteds, denoted by X. Their model reads [3]

$$
\begin{aligned}
\frac{d S}{d t} & =-\alpha S I-\kappa_{0} S \\
\frac{d I}{d t} & =+\alpha S I-\beta I-\kappa_{0} I-\kappa I \\
\frac{d R}{d t} & =+\beta I+\kappa_{0} S \\
\frac{d X}{d t} & =+\kappa I+\kappa_{0} I
\end{aligned}
$$

where, for the convenience of our analysis we set $S+I+R+X=1$, since the sum of Eqs. (1), (2), (3) and (4) is zero for any time $t$. In this model, the parameters $\alpha$ and $\beta$ are respectively the transmission rate and the recovery rate of the standard SIR model. In addition to that, and referring to the processes introduced by by Maier and Brockmann [3]: (1), the impact of containment efforts is captured by the terms proportional to the containment rate $\kappa_{0}$ that is effective in both $I$ and $S$ compartments, because non-pharmacological interventions such as social distancing and lock down affect the whole population alike; and (2), (symptomatic) infected individuals are removed at rate $\kappa$ corresponding to quarantine measures. By introducing this model, besides valuable insights on containment strategies to fight against COVID-19 and other possible similar infectious diseases outbreaks, Maier and Brockmann [3] were able to explain the subexponential growth in the number of confirmed COVID-19 cases in China. Their analysis and fittings reproduce empirical case counts in all provinces for plausible parameter values, suggesting that quarantine and containment shielded the susceptible population from the transmission process. By further analyzing their model, our objective here is different, though.

Since the adoption of non-pharmacological interventions previously described as (1) containment and (2) quarantine have a huge impact on many aspects in daily life, the use of such health public strategies is quite complex. To properly manage them, it is crucial to have at least one decision criterion to define when (and 
how) to start and stop these interventions. In order to address this issue, first we suppose that the health system has the capacity of attending $I_{M}$ infected individuals (being just a part of them WITH severe disease). When $I(t)>I_{M}$, then we say that there is a collapse in the health system. Based on that, we define our threshold criterion for switching between both containment and quarantine application and the "do nothing" strategy. Given a certain fraction threshold of infecteds $I_{m}$, with $I_{m}<I_{M}$, our control protocol is defined as:

- If $I(t)>I_{m}$, containment and quarantine are switched on (i.e., SIR-X dynamics);

- If $I(t)<I_{m}$, containment and quarantine are switched off (i.e., SIR dynamics).

Since $R$ and $X$ do not affect equations (1)-(2), we reduce the dynamic analysis to the plane $(S, I)$. But before this discussion, let us present how this paper is organized. In Sect. 2, we present some concise remarks on piecewise smooth dynamical systems. In Sect. 3, we define our piecewise smooth non-pharmacological intervention protocol for COVID-19. In Sect. 4, we establish the results of our main analysis, and in Sect. 5 some criteria about the best rate of containment effort are given. Finally, Sect. 6 closes the paper with some concluding remarks.

\section{Remarks on piecewise smooth dynamical systems}

Consider the smooth scalar function $h: \mathcal{D} \subset \mathbb{R}^{2} \rightarrow$ $\mathbb{R}$. The switching manifold of this system is defined by $\Sigma=\left\{(S, I) \in \mathcal{D}: h^{-1}(0)\right\}$. Clearly, $\Sigma$ is the separating boundary of the regions $R^{-}=\{\mathbf{x} \in \mathcal{D}$ : $h(\mathbf{x})<0\}$ and $R^{+}=\{\mathbf{x} \in \mathcal{D}: h(\mathbf{x})>0\}$.

Let be $\mathfrak{X}^{r}$ the space of $C^{r}$-vector fields on $\mathcal{D}$ endowed with the $C^{r}$-topology with $r=\infty$ or $r \geq 1$ large enough for our purposes and call $\Omega^{r}$ the space of vector fields $\mathbf{F}: \mathcal{D} \rightarrow \mathbb{R}^{2}$ such that

$\mathbf{F}(\mathbf{x})= \begin{cases}\mathbf{F}^{+}(\mathbf{x}), & \text { for } \mathbf{x} \in R^{+}, \\ \mathbf{F}^{-}(\mathbf{x}), & \text { for } \mathbf{x} \in R^{-},\end{cases}$

where $\mathbf{x} \in \mathcal{D}, \mathbf{F}^{ \pm} \in \mathfrak{X}^{r}$. We may consider $\Omega^{r}=\mathfrak{X}^{r} \times$ $\mathfrak{X}^{r}$ endowed with the product topology and denote any element in $\Omega^{r}$ by $\mathbf{F}=\left(\mathbf{F}^{+}, \mathbf{F}^{-}\right)$, which we will accept to be multivalued in points of $\Sigma$.

The contact of smooth vector fields $\mathbf{F}^{ \pm} \in \mathfrak{X}^{r}$ with $\Sigma$ are provided by the directional Lie derivatives:
$L_{\mathbf{F}^{ \pm}} h=\left\langle\nabla h, \mathbf{F}^{ \pm}\right\rangle$, where $\nabla h$ and $\langle\cdot, \cdot\rangle$ denote the gradient of smooth function $h$ and the canonical inner product, respectively. The higher order Lie derivatives are given by $L_{\mathbf{F}^{ \pm}}^{m} h=\left\langle\nabla L_{\mathbf{F}^{ \pm}}^{m-1} h, \mathbf{F}^{ \pm}\right\rangle$.

On $\Sigma$, we distinguish the following regions: (i) attractive sliding, (ii) repulsive sliding (or escape) and (iii) crossing, given by (i) $\Sigma_{a s}=\left\{\mathbf{x} \in \Sigma: L_{\mathbf{F}^{+}} h(\mathbf{x})<\right.$ $\left.0<L_{\mathbf{F}^{-}} h(\mathbf{x})\right\}$, (ii) $\Sigma_{r s}=\left\{\mathbf{x} \in \Sigma: L_{\mathbf{F}^{-}} h(\mathbf{x})<\right.$ $\left.0<L_{\mathbf{F}^{+}} h(\mathbf{x})\right\}$ and (iii) $\Sigma_{c}^{-}=\left\{\mathbf{x} \in \Sigma: L_{\mathbf{F}^{-}} h(\mathbf{x})<\right.$ 0 and $\left.L_{\mathbf{F}^{+}} h(\mathbf{x})<0\right\}, \Sigma_{c}^{+}=\left\{\mathbf{x} \in \Sigma: L_{\mathbf{F}^{-}} h(\mathbf{x})>\right.$ 0 and $L_{\mathbf{F}^{+}} h(\mathbf{x})>0$ \}. As we will see later, Figure 2 presents regions $\Sigma_{a s}, \Sigma_{c}^{-}$and $\Sigma_{c}^{+}$.

In this paper, we suppose that the sliding dynamics in $\Sigma_{a s} \cup \Sigma_{r s}$ is described by the Filippov sliding vector field $[9,10]$ defined as

$\mathbf{F}^{s}(\mathbf{x})=(1-\lambda) \mathbf{F}^{-}(\mathbf{x})+\lambda \mathbf{F}^{+}(\mathbf{x})$,

where for each $\mathbf{x} \in \Sigma_{a s} \cup \Sigma_{r s}$, the value of $\lambda$ is chosen such that $L_{\mathbf{F}_{s}} h(\mathbf{x})=0$, i.e.,

$\lambda=\lambda(\mathbf{x})=\frac{L_{\mathbf{F}^{-}} h(\mathbf{x})}{L_{\mathbf{F}^{-}} h(\mathbf{x})-L_{\mathbf{F}^{+}} h(\mathbf{x})}$,

provided that the denominator of the previous expression does not vanish. Therefore, $\lambda \in(0,1)$ for all $\mathbf{x} \in \Sigma_{a s} \cup \Sigma_{r s}$, while $\lambda=0$ implies that $L_{\mathbf{F}^{-}} h(\mathbf{x})=0$, i.e., $\mathbf{x}$ is a tangency point of the vector field $\mathbf{F}^{-}$with the boundary $\Sigma$, and $\lambda=1$ implies that $L_{\mathbf{F}^{+}} h(\mathbf{x})=0$, i.e., $\mathbf{x}$ is a tangency point of the vector field $\mathbf{F}^{+}$with the boundary $\Sigma$.

The orbits of the system (5) can be constructed by concatenating standard solutions in $R^{ \pm}$and sliding solutions on $\Sigma$ following the sliding system $\dot{\mathbf{x}}=\mathbf{F}^{s}(\mathbf{x})$. For more details, the reader is referred to the reference [9]. The forward orbit of (5) that crosses $\Sigma$ goes from $R^{-}$to $R^{+}$through $\mathbf{x}_{0} \in \Sigma_{c}^{+}$and goes from $R^{+}$to $R^{-}$ through $\mathbf{x}_{0} \in \Sigma_{c}^{-}$. The forward orbit of (5) that intersects $\Sigma$ at a point $\mathbf{x}_{0} \in \Sigma_{a s}$ continues from this point $\mathbf{x}_{0}$ on a contained sliding motion in $\Sigma$.

Sliding regions are bound by points where the vector fields $\mathbf{F}^{ \pm}$are tangent to $\Sigma$. Then, we define two sets of tangential singularities:

$$
\begin{aligned}
& T_{+}=\left\{\mathbf{x} \in \Sigma: L_{\mathbf{F}^{+}} h(\mathbf{x})=0\right\} \\
& \text { and } T_{-}=\left\{\mathbf{x} \in \Sigma: L_{\mathbf{F}^{-}} h(\mathbf{x})=0\right\} ;
\end{aligned}
$$

one for each vector field. In the following, we provide the definitions of tangential singularities $[10,11]$.

Definition 1 One says that $\mathbf{x} \in \Sigma$ is a fold point of $\mathbf{F}^{+}$ (respectively, $\mathbf{F}^{-}$) if $L_{\mathbf{F}^{+}} h(\mathbf{x})=0$ and $L_{\mathbf{F}^{+}}^{2} h(\mathbf{x}) \neq 0$ 
(respectively, $L_{\mathbf{F}^{-}} h(\mathbf{x})=0$ and $L_{\mathbf{F}^{-}}^{2} h(\mathbf{x}) \neq 0$ ). Moreover, we say that this fold point is visible if $L_{\mathbf{F}^{+}}^{2} h(\mathbf{x})>$ 0 or invisible if $L_{\mathbf{F}^{+}}^{2} h(\mathbf{x})<0$ (respectively, $L_{\mathbf{F}^{-}}^{2} h(\mathbf{x})<$ 0 or invisible if $L_{\mathbf{F}^{-}}^{2} h(\mathbf{x})>0$ ).

Observe that system (5) can have equilibria for each vector field $\mathbf{F}^{ \pm}$and also equilibria for the sliding vector field $\mathbf{F}^{s}$.

\section{A piecewise smooth non-pharmacological protocol for COVID-19}

Note that, in order to study the dynamic of the SIR$\mathrm{X}$ model, it is sufficient to consider equations (1)-(2). This is possible because such equations are written only in terms of the state variables $S$ and $I$. Moreover, to ensure that the health system will not collapse, we control the fraction of infected individuals such that $I(t)$ cannot be over $I_{M}$. In what follows, we consider the two-dimensional dynamical system (1)-(2) defined in the set

$\mathcal{D}=\left\{(S, I) \in \mathbb{R}^{2}: 0 \leq S \leq 1-I, 0 \leq I \leq I_{M}\right\}$

and $h$ given by $h(S, I)=I-I_{m}$.

As we mentioned before, we set $\kappa=\kappa_{0}=0$ for $I<I_{m}$ and $\kappa_{0}>0$ and $\kappa>0$ as constants for $I>I_{m}$, where $I_{m}$ is a design parameter such that $0<I_{m}<I_{M}$; in fact, $I_{m}$ is the fraction of infected individuals below which containment and quarantine actions are null and above which such actions are applied at rates $\kappa_{0}>0$ and $\kappa>0$. In addition to that, we use the Filippov's convention to determine what happens when $I=I_{m}$.

In the piecewise smooth vector field framework, we rewrite the system (1)-(2) as

$\left[\begin{array}{l}\frac{d S}{d t} \\ \frac{d I}{d t}\end{array}\right]= \begin{cases}\mathbf{F}^{-}(S, I), & \text { if } I<I_{m}, \\ \mathbf{F}^{+}(S, I), & \text { if } I>I_{m},\end{cases}$

composed by the vector fields

$$
\begin{gathered}
\mathbf{F}^{-}(S, I)=\left[\begin{array}{c}
-\alpha I S \\
\alpha\left(S-\frac{1}{R_{0, \text { free }}}\right) I
\end{array}\right] \\
\text { and } \quad \mathbf{F}^{+}(S, I)=\left[\begin{array}{c}
-\left(\alpha I+\kappa_{0}\right) S \\
\alpha\left(S-\frac{1}{R_{0, \text { eff }}}\right) I
\end{array}\right],
\end{gathered}
$$

where $(S, I) \in \mathcal{D}$ and

$R_{0, \text { free }}=\frac{\alpha}{\beta}>R_{0, \text { eff }}=\frac{\alpha}{\beta+\kappa+\kappa_{0}}$.
The vector field $\mathbf{F}^{-}$has a line of equilibria points at $I=0$ and $0 \leq S \leq 1$, being attractive in the part where $S<\frac{1}{R_{0, \text { free }}}$, while $\mathbf{F}^{+}$has a virtual stable node equilibrium at $(0,0)$, i.e., it is placed in $R^{-}$where $\mathbf{F}^{+}$ is not defined. See Fig. 1. From the first Lie derivatives, given by $L_{\mathbf{F}^{-}} h\left(S, I_{m}\right)=\alpha\left(S-\frac{1}{R_{0, \text { free }}}\right) I_{m}$ and $L_{\mathbf{F}^{+}} h\left(S, I_{m}\right)=\alpha\left(S-\frac{1}{R_{0, \text { eff }}}\right) I_{m}$, it is easy to see that there are two tangency points in $\Sigma$, namely

$\mathbf{q}^{-}=\left(\frac{1}{R_{0, \text { free }}}, I_{m}\right) \quad$ and $\quad \mathbf{q}^{+}=\left(\frac{1}{R_{0, \text { eff }}}, I_{m}\right)$.

Specifically, $\mathbf{q}^{-}$is a visible fold for $\mathbf{F}^{-}$and $\mathbf{q}^{+}$is an invisible fold for $\mathbf{F}^{+}$, since $L_{\mathbf{F}^{-}}^{2} h\left(\mathbf{q}^{-}\right)=-\frac{\alpha^{2}}{R_{0, \text { free }}} I_{m}^{2}<$ 0 and $L_{\mathbf{F}^{+}}^{2} h\left(\mathbf{q}^{+}\right)=-\frac{\alpha I_{m}}{R_{0, \text { eff }}}\left(\alpha I_{m}+\kappa_{0}\right)<0$. Completing $\Sigma$, we have an attractive sliding segment defined by

$\Sigma_{a s}=\left\{(S, I) \in \Sigma: I=I_{m}, \quad \frac{1}{R_{0, \text { free }}}<S<\frac{1}{R_{0, \text { eff }}}\right\}$,

with extremes at the fold points. The crossing regions are given by two half straight lines:

$$
\begin{aligned}
& \Sigma_{c}^{+}=\left\{(S, I) \in \Sigma: I=I_{m}, \quad S>\frac{1}{R_{0, \mathrm{eff}}}\right\} \\
& \text { and } \quad \Sigma_{c}^{-}=\left\{(S, I) \in \Sigma: I=I_{m}, \quad S<\frac{1}{R_{0, \text { free }}}\right\} .
\end{aligned}
$$

The sliding dynamics in $\Sigma_{a s}$ is governed by the sliding vector field $\mathbf{F}_{S}(S, I)=\left(f_{S}(S, I), 0\right)$, where

$$
f_{S}(S, I)=\alpha\left(\frac{\kappa_{0}}{\kappa_{0}+\kappa} \frac{1}{R_{0, \text { free }}}-I\right) S-\frac{\kappa_{0}}{\kappa_{0}+\kappa} S^{2} .
$$

\section{Main analysis}

In this section, we present our main analysis of this paper about the non-pharmacological strategies of control of the COVID-19. We emphasize that the analysis presented here is global, i.e.,

we establish the dynamic for all initial conditions on model domain.

In practice, it is almost impossible to determine the real initial condition in order to apply some evaluation process. This being, we eliminate the dependence on the initial condition and we describe the dynamic for all $\left(S_{0}, I_{0}\right) \in \mathcal{D}$, defined in (6). 
4.1 Global analysis and asymptotic stability

Proposition 1 Consider the system (7) defined in $\mathcal{D}$. The following statements hold.

(a) If $R_{0, \text { free }}<\frac{1}{1-I_{m}}$ then on the switching manifold $\Sigma$ coincides with the crossing mode $\Sigma_{c}^{-}$. Besides, if $R_{0, \text { free }}=\frac{1}{1-I_{m}}$ then the visible fold $\mathbf{q}^{-}=(1-$ $\left.I_{m}, I_{m}\right) \in \Sigma$, see Fig. $2 a$.

(b) If $R_{0, \text { eff }} \leq \frac{1}{1-I_{m}}<R_{0, \text { free }}$ then on the switching manifold there are the $\Sigma_{c}^{-}$crossing mode and the $\Sigma_{\text {as }}$ sliding mode, separated by the visible fold $\mathbf{q}^{-}$. Besides, if $R_{0, \text { eff }}=\frac{1}{1-I_{m}}$ then the invisible fold $\mathbf{q}^{+}=\left(1-I_{m}, I_{m}\right) \in \Sigma$, see Fig. $2 b$.

(c) If $R_{0, \text { eff }}>\frac{1}{1-I_{m}}$ then the switching manifold presents the crossing modes $\Sigma_{c}^{-}$and $\Sigma_{c}^{+}$, the sliding mode $\Sigma_{a s}$, the visible fold $\mathbf{q}^{-}$and the invisible fold $\mathbf{q}^{+}$, see Fig. $2 c-f$.

Proof It follows by direct calculations of Lie's derivatives of the system (7).

The next results provide the global stability of the model. Moreover, it establishes when it is possible to have trajectories leaving the set $\mathcal{D}$, collapsing the health system.

Theorem 1 Consider the systems (7) defined in D. The following statements hold:

(a) The straight line segment $\Gamma=\{(S, 0) \in \mathcal{D}: 0 \leq$ $\left.S<\frac{1}{R_{0, \text { free }}}\right\}$ is globally asymptotically stable. Moreover, it is composed by (non-hyperbolic) equilibria.

(b) When $\Sigma_{a s} \neq \varnothing$, every trajectory that colides to $\Sigma_{a s}$ leaves this set after a finite time and enters in the region

$$
\begin{gathered}
\mathcal{A}=\left\{(S, I) \in \mathcal{D}: 0 \leq S<\frac{1}{R_{0, \text { free }}}\right. \\
\text { and } \left.0 \leq I<I_{m}\right\} .
\end{gathered}
$$

(c) If $R_{0, \text { eff }} \leq \frac{1}{1-I_{M}}$ then $(S(t), I(t)) \in \mathcal{D}$ for all $t>t_{0}$ and $\left(S_{0}, I_{0}\right) \in \mathcal{D}$.

Proof (a) Putting $I=0$ in (7), we conclude that the $S$-axis is composed by equilibria. Moreover, following the analysis done in Proposition 1, all trajectories starting in a point in $\mathcal{D}$ enter $\mathcal{A}$ and then converge to $\Gamma$.

(b) By (9), we get that

$$
\begin{aligned}
f_{s}\left(S, I_{m}\right)= & \left(\frac{\kappa_{0}}{\kappa_{0}+\kappa} \frac{1}{R_{0, \text { free }}}-I_{m}\right) S \\
& -\frac{\kappa_{0}}{\kappa_{0}+\kappa} S^{2}<0,
\end{aligned}
$$

because $S>\frac{1}{R_{0, \text { free }}}>\frac{1}{R_{0, \text { free }}}-\frac{\kappa_{0}}{\kappa_{0}+\kappa} I_{m}$. Then, the $\mathbf{F}_{S}$ is negative for all $(S, I) \in \Sigma_{a s}$. This means that the system trajectory that touches $\Sigma$ at a point of $\Sigma_{a s}$, remains sliding in $\Sigma_{a s}$ tending to the visible fold point $\mathbf{q}^{-}$in finite time. When the trajectory reaches $\mathbf{q}^{-}$, it leaves $\Sigma$ and enters the region below $\Sigma$ whose dynamics is governed by $\mathbf{F}^{-}$.

(c) Note the vector fields $\mathbf{F}^{ \pm}$on the border of $\mathcal{D}$; see Figs. 1 and 2. On the boundaries (i) $S=0$, (ii) $I=0$, (iii) $g(S, I)=S+I=1$ and (iv) $I=I_{M}$, $0<S \leq 1-I_{M}$, we have: (i) $\frac{d S}{d t}=0$ and $\frac{d I}{d t}<0$ for both vector fields; (ii) $\frac{d S}{d t}=\frac{d I}{d t}=0$ for $\mathbf{F}^{-}$; (iii) $\frac{d g}{d t}<0$ for both vector fields; and (iv) for $\mathbf{F}^{+}, \frac{d I}{d t}=$ $\alpha\left(S-\frac{1}{R_{0, \text { eff }}}\right) I<0$ whenever $R_{0, \text { eff }}<\frac{1}{1-I_{M}}$, and $\frac{d I}{d t}=0, \frac{d^{2} I}{d t^{2}}<0$ when $R_{0, \text { eff }}=\frac{1}{1-I_{M}}$.

\subsection{Global bifurcation analysis}

In Fig. 1a we present the $\left(R_{0, \text { free }}, R_{0, \text { eff }}\right)$-plane of parameters, from where we get four main configurations of the vector fields $\mathbf{F}^{ \pm}$, shown in Fig. $1 \mathrm{~b}$.

In summary:

(a) $\Gamma=\left\{(S, 0) \in \mathcal{D}: 0 \leq S<\frac{1}{R_{0, \text { free }}}\right\}$ is the global attractor.

(b) For $R_{0 \text {,free }} \leq \frac{1}{1-I_{m}}$ (region 1 ), every trajectory started below $\Sigma$ remains below $\Sigma$ tending to $\Gamma$.

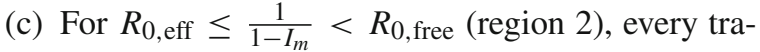
jectory started below $\Sigma$ remains below $\Sigma$ or reaches $\Sigma$ at the $\Sigma_{a s}$ part, and from there it slides on $\Sigma$ and returns to below $\Sigma$ after a finite time. Moreover, there is a trajectory that tangentially touches $\Sigma$ at $\mathbf{q}^{-}$.

(d) The situation described in (c) also happens for $\frac{1}{1-I_{m}}<R_{0, \text { eff }} \leq \frac{1}{1-I_{M}}$ (region 3). In addition, though, there are also trajectories starting below $\Sigma$ that cross $\Sigma$ passing to the region above $\Sigma$. However, the invisible characteristic of the tangent fold point of the vector field $\mathbf{F}^{+}$ensures that this trajectory returns to $\Sigma$ at a point of $\Sigma_{c}^{-}$or $\Sigma_{a s}$, or still at $\mathbf{q}^{-}$. Again, every trajectory with initial condition $\left(S\left(t_{0}\right), I\left(t_{0}\right)\right) \in \mathcal{D}$ remains in $\mathcal{D}$ for all $t>t_{0}$ and still tends to $\Gamma$ as $t \rightarrow \infty$.

(e) For $R_{0, \text { eff }}>\frac{1}{1-I_{M}}$ (region 4 ), there is a subset of $\mathcal{D}$ in which the system trajectories leave $\mathcal{D}$ after a positive time. 


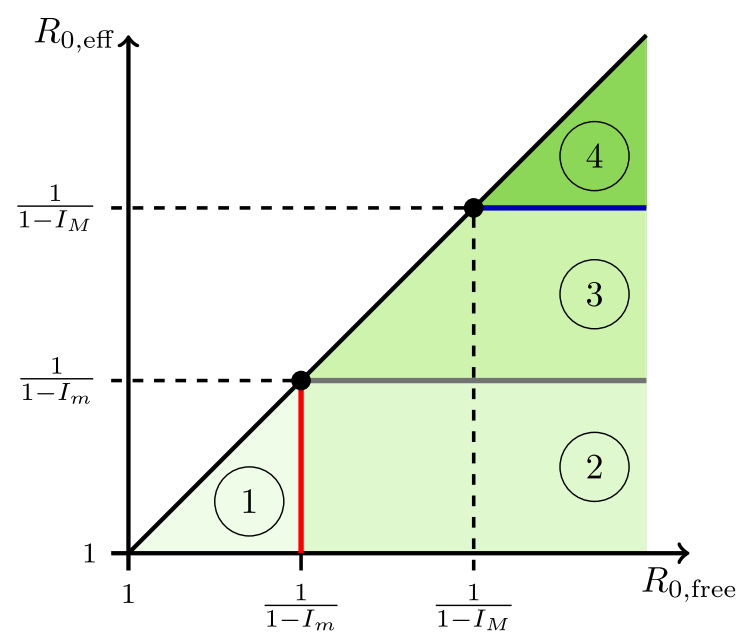

(a) Bifurcation set
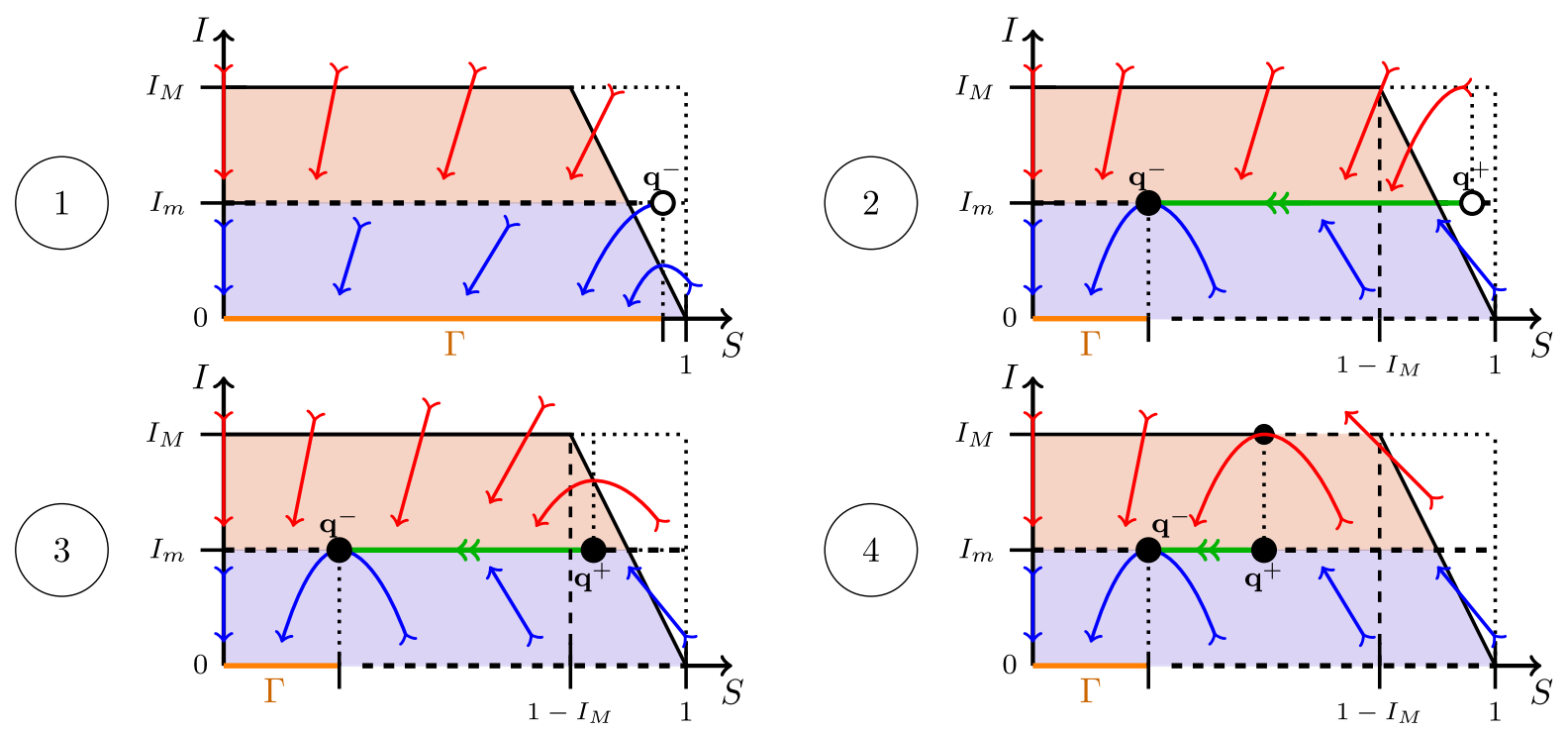

(b) Dynamics in $\mathcal{D}$

Fig. 1 Transition of the dynamics of the system (7) in $\mathcal{D}$, from the variation of parameters $R_{0}$, free and $R_{0 \text {,eff }}$

At the transition points between scenarios 1 and 2 (red line), 2 and 3 (gray line), predicted by the bifurcation set of Fig. 1(a), the phase portrait of the system (7) undergoes a bifurcation associated with appearance (or disappearance) of tangency points and also sliding and crossed segments in the domain $\mathcal{D}$.

The critical phase portrait at the transition between the scenarios 1 to 2 and from 2 to 3, are given in Fig. 2a and $\mathrm{b}$. A third bifurcation occurs at the transition from 3 to 4 (blue line), having the critical phase portrait like the one in Fig. 2c. From there, the domain $\mathcal{D}$ is no longer positively invariant to the flow of the system (7), leading to three sub-scenarios:

(a) Trajectories starting from $\Sigma \subset \mathcal{D}$, or below it, remain within $\mathcal{D}$ (Fig. 2d).

(b) The same as the previous one, but the trajectory starting at $\left(1-I_{m}, I_{m}\right) \in \Sigma$ is tangent to the upper threshold $I=I_{M}$ (Fig. 2e).

(c) The trajectory starting at $\left(1-I_{m}, I_{m}\right) \in \Sigma$ is transversal to the upper threshold $I=I_{M}$, and 
therefore one cannot guarantee that the system will not collapse for any initial condition below $\Sigma$ (Fig. 2f).

\section{The best rate of containment effort}

By using the results presented in Sect. 4, we can predict that the system will not collapse for any choice of $I_{m}<$ $I_{M}$ whenever $R_{0, \text { eff }} \leq \frac{1}{1-I_{M}}$, which is in accordance with statement (c) of Theorem 1. However, this may require high rates of confinement $\kappa_{0}$ and quarantine $\kappa$. To obtain lower rates without causing the system to collapse for any initial condition below the control threshold $I_{m}$, we must choose the value of $I_{m}$ properly. For this case, we have $R_{0, \text { free }}>R_{0, \text { eff }} \geq \frac{1}{1-I_{M}}$, as it is shown in region 4 of Fig. 1a.

In what follows, we consider $R_{0, \text { eff }} \geq \frac{1}{1-I_{M}}$ and under this assumption we determine the condition on the system parameters, in particular $I_{m}$ and $\kappa_{0}$, which ensures that any trajectory starting at $\mathcal{D}$ below $I=I_{m}$, follows within $\mathcal{D}$ for time forward. In this way,

we provide necessary and sufficient conditions for the control of COVID-19 ensuring the non-collapse of the health system but also minimizing the containment efforts.

Consider that $I_{0} \leq I_{m} \leq I_{M}$. In this context, two natural questions are:

Question 1: For a fixed $I_{m}$, what is the minimum containment rate $\kappa_{0}$ that ensures $I(t) \leq I_{M}$ ?

Question 2: For a fixed $\kappa_{0}$, what is the maximum value of infecteds $I_{m}$ that ensures $I(t) \leq I_{M}$ ?

Both questions are in fact equivalent and their answers correspond to the limit case illustrated in Fig. $2 \mathrm{e}$. The condition required for this is that the trajectory of the system (7) started at point $\left(1-I_{m}, I_{m}\right)$ passes through point $\left(1 / R_{0, \text { eff }}, 1-I_{M}\right)$. We assume that the parameters $I_{m}$ and $\kappa_{0}$ vary and the others parameters are fixed such that $R_{0, \text { free }}>R_{0, \text { eff }} \geq \frac{1}{1-I_{M}}$.

In the next proposition we explicitly exhibit the expression of the value of $\kappa_{0}$ that answer the Question 1 (or, equivalently, we present implicitly the value of $I_{m}$ that responds to the Question 2). More precisely, fixing $I_{m}$ and all the others parameters of the system (7) we present the minimum containment rate $\kappa_{0}$ that satisfies $I(t) \leq I_{M}$.
We define the set:

$$
\begin{aligned}
\mathcal{B}= & \left\{\left(I_{m}, \kappa_{0}\right) \in \mathbb{R}^{2}: 0<I_{m} \leq I_{M} \text { and } 0 \leq \kappa_{0}\right. \\
& \left.\leq \alpha\left(1-I_{M}\right)-\kappa-\frac{\alpha}{R_{0, \text { free }}}\right\}
\end{aligned}
$$

Proposition 2 We suppose that $\left(I_{m}, \kappa_{0}\right) \in \mathcal{B}$. Then the minimum containment rate $\kappa_{0}$ that guaranties that $I(t) \leq I_{M}$ is given by

$$
\begin{aligned}
\kappa_{0}= & \kappa_{0}^{\min }\left(I_{m}\right)=-\kappa-\frac{\alpha}{R_{0, \text { free }}} \\
& -\frac{\alpha\left(1-I_{M}+\left(\frac{1}{R_{0, \text { free }}}+\frac{\kappa}{\alpha}\right) \log \left[\frac{I_{M}}{I_{m}}\right]\right)}{\text { ProductLog }\left[-1,\left(1-I_{M}+\left(\frac{1}{R_{0, \text { free }}}+\frac{\kappa}{\alpha}\right) \log \left[\frac{I_{M}}{I_{m}}\right]\right) \Psi\right]},
\end{aligned}
$$

where

$\Psi=-\frac{1}{1-I_{m}}\left(\frac{I_{m}}{I_{M} e^{1-I_{m}\left(1+\log \left[\frac{I_{M}}{I_{m}}\right]\right)}}\right)^{\frac{1}{1-I_{m}}}$.

Proof Let $\left(S_{0}, I_{0}\right) \in \mathcal{D}$ be the initial condition for the system (7). Assuming $I_{0} \geq I_{m}$, in the $(S, I)$-plane, a first integral for the system $\mathbf{F}^{+}$is given by

$$
\begin{gathered}
\bar{\sigma}\left(S, S_{0}, I, I_{0}\right)=S-S_{0}+I-I_{0} \\
-\frac{1}{R_{0, \text { eff }}} \log \left[\frac{S}{S_{0}}\right]+\frac{\kappa_{0}}{\alpha} \log \left[\frac{I}{I_{0}}\right] .
\end{gathered}
$$

To obtain the condition under the parameter $\kappa_{0}$, such that the orbit started at point $\left(1-I_{m}, I_{m}\right)$ passes through the point $\left(1 / R_{0, \text { eff }}, 1-I_{M}\right)$ it is sufficient to consider

$$
\begin{aligned}
& \sigma\left(I_{m}, \kappa_{0}\right) \\
& =\bar{\sigma}\left(1 / R_{0, \mathrm{eff}}, 1-I_{m}, I_{M}, I_{m}\right) \\
& =\frac{\kappa_{0}}{\alpha} \log \left(\frac{I_{M}}{I_{m}}\right) \\
& \quad+\frac{1}{R_{0, \mathrm{eff}}\left(\kappa_{0}\right)} \log \left(\left(1-I_{m}\right) R_{0, \mathrm{eff}}\left(\kappa_{0}\right)\right) \\
& \quad+\frac{1}{R_{0, \mathrm{eff}}\left(\kappa_{0}\right)}+I_{M}-1=0 .
\end{aligned}
$$

Note that the parameters $\kappa_{0}$ and $R_{0, \text { eff }}$ satisfy the relations:

$$
\begin{aligned}
& \text { If } \kappa_{0}<\alpha\left(1-I_{M}\right)-\kappa-\frac{\alpha}{R_{0, \text { free }}} \text { then } R_{0, \text { eff }} \\
& >\frac{1}{1-I_{M}} . \\
& \text { If } \kappa_{0}=\alpha\left(1-I_{M}\right)-\kappa-\frac{\alpha}{R_{0, \text { free }}} \text { then } R_{0, \text { eff }} \\
& =\frac{1}{1-I_{M}} .
\end{aligned}
$$


Fig. 2 Phase portraits of the system (7). In a we see the dynamics of the transition scenario from region 1 to 2 of the bifurcation set shown in Fig. 1. In $\mathbf{b}$ we see the dynamics of the transition scenario from region 2 to 3 . And in c, the transition scenario from region 3 to 4 . In $\mathbf{d}-\mathbf{f}$ are shown the dynamics for $\left(R_{0, \text { free }}, R_{0, \text { eff }}\right)$ in the region 4 , in which the system trajectory starting at $\left(1-I_{m}, I_{m}\right)$ is tangent to the upper threshold $I=I_{M}$ when $\sigma\left(I_{m}, \kappa_{0}\right)=0$, as shown in $\mathbf{e}$

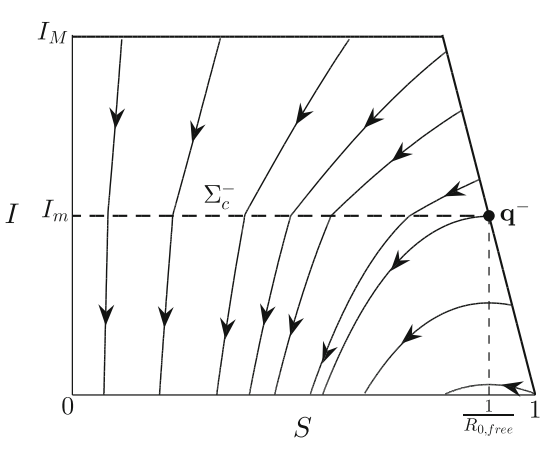

(a) For $R_{0, \text { free }}=\frac{1}{1-I_{m}}$.

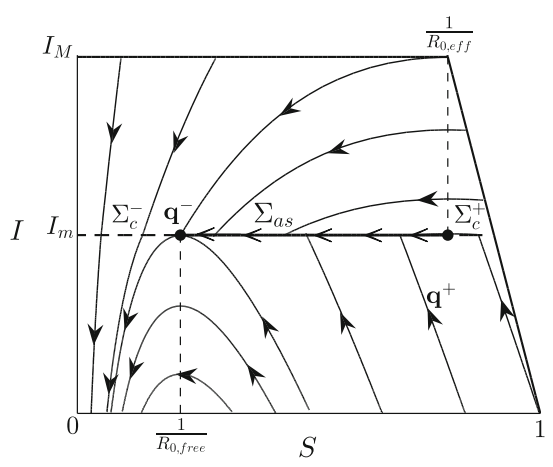

(c) For $R_{0, \mathrm{eff}}=\frac{1}{1-I_{M}}$.

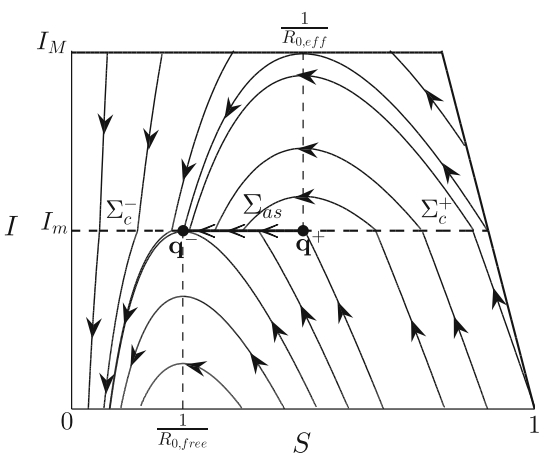

(e) For $R_{0, \text { eff }}>\frac{1}{1-I_{M}}$ and $\sigma=0$.

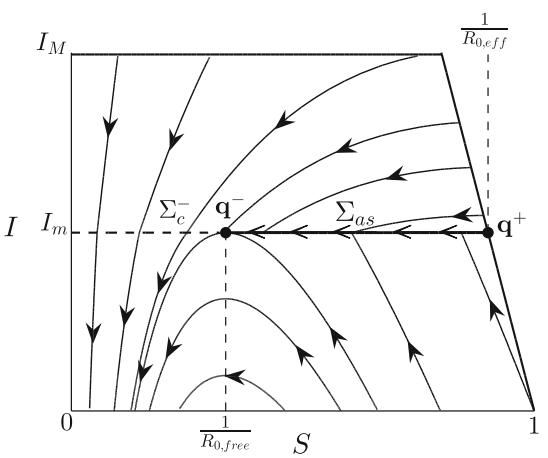

(b) For $R_{0, \text { eff }}=\frac{1}{1-I_{m}}$.

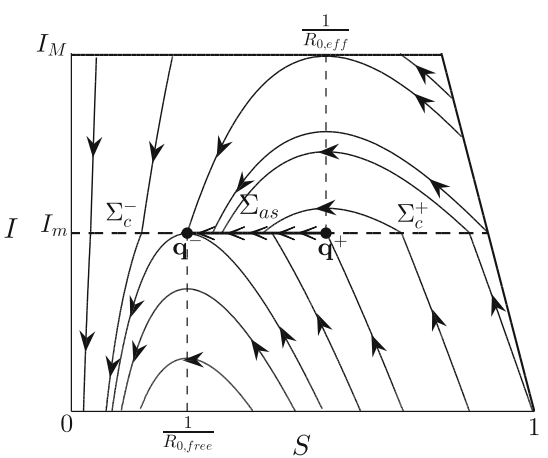

(d) For $R_{0, \text { eff }}>\frac{1}{1-I_{M}}$ and $\sigma>0$.

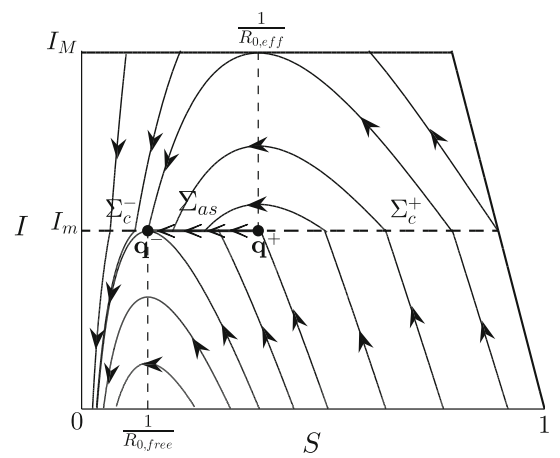

(f) For $R_{0, \text { eff }}>\frac{1}{1-I_{M}}$ and $\sigma<0$.
Besides then, remembering that $\frac{1}{R_{0, \mathrm{eff}}}=\frac{1}{R_{0, \text { free }}}+\frac{\kappa+\kappa_{0}}{\alpha}$, we get that

$$
\begin{aligned}
\nabla \sigma & \left(I_{m}, \kappa_{0}\right) \\
= & \left(-\frac{\kappa_{0}}{\alpha I_{m}}-\frac{1}{\left(1-I_{m}\right) R_{0, \mathrm{eff}}}, \frac{1}{\alpha} \log \left(\frac{I_{M}}{I_{m}}\right)\right. \\
& \left.+\frac{1}{\alpha} \log \left(\left(1-I_{m}\right) R_{0, \mathrm{eff}}\right)\right) \neq(0,0) .
\end{aligned}
$$

In this way, by the Implicit Function Theorem, it is possible solve the equation $\sigma\left(I_{m}, \kappa_{0}\right)=0$ in terms of $\kappa_{0}$ or $I_{m}$. Therefore, solving this equation in terms of $\kappa_{0}$, we obtain the expression given in (10).
The curve in the $\left(I_{m}, \kappa_{0}\right)$-plane given by $\sigma\left(I_{m}, \kappa_{0}\right)=$ 0 , see Fig. 3, contains all the solutions for Questions 1 and 2 . In this case, if $\left(\widehat{I}_{m}, \widehat{\kappa}_{0}\right)$ is a solution of $\sigma\left(I_{m}, \kappa_{0}\right)=0$ in domain $\mathcal{B}$, then, for $\kappa_{0}=\widehat{\kappa}_{0}$ fixed the maximum $I_{m}$ is $\widehat{I}_{m}$ and for $I_{m}=\widehat{I}_{m}$ fixed the minimum $\kappa_{0}$ is $\widehat{\kappa}_{0}$.

The solution branch of $\sigma\left(I_{m}, \kappa_{0}\right)=0$ is represented numerically in a $\left(I_{m}, \kappa_{0}\right)$-parameter plane, as shown in Fig. 3, for three different $\kappa$ values and from which we observe two different situations separated by the critical 


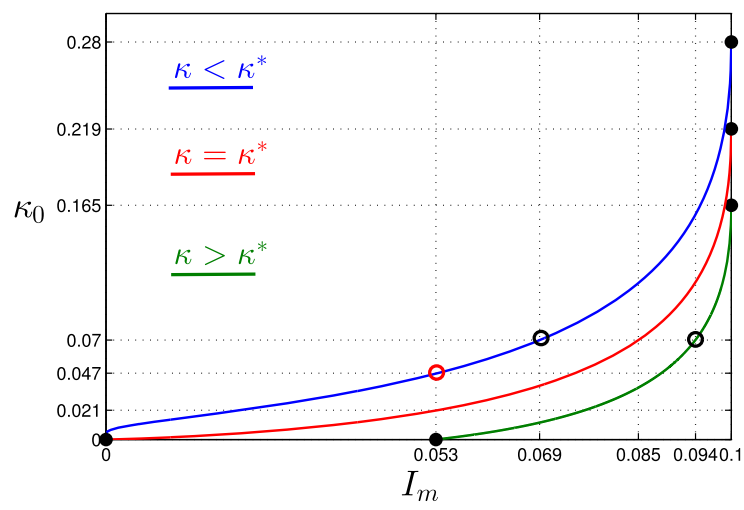

Fig. 3 Graphs of $\sigma\left(I_{m}, \kappa_{0}\right)=0$ for the parameters $\alpha=0.7$, $R_{0, \text { free }}=4.6667, I_{M}=0.1, \kappa=0.2$ (blue), $\kappa=\kappa^{*}=0.2613$ (red) and $\kappa=0.315$ (green)

value

$\kappa=\kappa^{*}=\alpha e^{1+\text { ProductLog }\left[-1,\left(I_{M}-1\right) e^{-1}\right]}-\frac{\alpha}{R_{0, \text { free }}}$,

as described in the sequel.

(a) If $\kappa>\kappa^{*}$, then the solution branch of $\sigma\left(I_{m}, \kappa_{0}\right)=$ 0 cuts the $I_{m}$-axis at $I_{m}>0$. In this case, for any $I_{m}$ such that

$$
\begin{gathered}
I_{m} \leq I_{m}^{*}(\kappa)=1-\left(\frac{1}{R_{0, \text { free }}}+\frac{\kappa}{\alpha}\right) \\
e^{-1+\frac{\left(1-I_{M}\right) \alpha R_{0, \text { free }}}{\alpha+\kappa R_{0, \text { free }}}},
\end{gathered}
$$

the minimum containment rate is $\kappa_{0}^{\min }\left(I_{m}\right)=0$. Naturally, for $\kappa_{0}=0$ the maximum of $I_{m}$ is $I_{m}^{\max }(0)=I_{m}^{*}$. This means that, considering a quarantine rate greater than $\kappa^{*}$, the containment effort could be even zero if the quarantine strategies are implemented before the fraction of infected individuals attains the value $I_{m}^{*}$. In this case, the strategy could reach just the infected individuals and none restriction is applied to the general population.

(b) If $\kappa \leq \kappa^{*}$, then the solution branch of $\sigma\left(I_{m}, \kappa_{0}\right)=$ 0 does not cut the $I_{m}$-axis at $I_{m}>0$ and

$$
\lim _{I_{m} \rightarrow 0} \kappa_{0}^{\min }\left(I_{m}\right)=0
$$

This means that even not considering a large quarantine rate, the minimum containment effort converges to zero only if the containment strategies are implemented very soon.

In order to exemplify our theoretical results, let us consider an example for selected parameter values.
Example 1 Let $\alpha=0.7, R_{0, \text { free }}=4.6667, I_{M}=0.1$ and $\kappa \in[0.2,0.315]$.

(a) If $\kappa_{0}=0.07$, what is the maximum value for $I_{m}$ that ensures $I(t) \leq I_{M}$ ? In Fig. 3 observe that for $\kappa=0.2$ we have $I_{m}^{\max }=0.069$, and for $\kappa=0.315$ we have $I_{m}^{\max }=0.094$ (these points are marked with black circles). In addition, for $\kappa=0.315>$ $\kappa^{*}=0.2613$ we can take $\kappa_{0}=0$ if control actions are used earlier. This is possible if we choose some $I_{m} \leq I_{m}^{*}=0.053$.

(b) Taking $I_{m}=0.053$ and $\kappa=0.2$, then the minimum value of $\kappa_{0}$ that ensures $I(t) \leq I_{M}$ is $\kappa_{0}^{\min }=0.04667$ (the red circle marks this point in Fig. 3). However, for $\kappa \geq 0.315$ we have $\kappa_{0}^{\min }=0$.

Figure 4 shows two simulation results of the system (1)-(4) under the assumed control protocol. We take $I_{m}=0.05<I_{m}^{*}=0.053$ and the other parameters as in the previous example. For the first case, which is shown in Fig. $4 \mathrm{a}-4 \mathrm{~b}$, we use $\kappa_{0}=0.07$ and $\kappa=0.2$. Thus, the containment measures are maintained for a period of about 15 days (from day 15 to day 30). For the second case, which is shown in $4 \mathrm{c}-\mathrm{d}$, we use $\kappa_{0}=0$ and $\kappa=0.315$. For this choice, the period of the containment action is longer than the previous one, being about 35 days (from day 15 to day 50). In these simulations, we consider a hysteresis band, defined by $\delta>0$ and below $I=I_{m}$, in order to limit the switching frequency and to avoid the chattering phenomenon at the switching boundary $I=I_{m}$. By adjusting the parameter $\delta$, we can reduce the maximum switching frequency of the sliding motion, in addition to decreasing the average fraction of infected during the period of application of the containment. The global dynamics results under the constraint $I(t)<I_{M}$ are preserved regardless of the choice of $\delta$. In both cases reported in Fig. 4, and also in general, the fraction of individuals $R(t)$ and $X(t)$ depends solely on $S(t)$ and on $I(t)$. Moreover, from the epidemiological point of view, susceptibles and infected are the essential relevant compartments. Based on these reasons, we present our analysis only in terms of $S(t)$ and $I(t)$. As a last comment, though, Fig. 4 shows the evolution of all variables. In both cases, after stopping the control measures, the system evolves according to the SIR dynamics and then $X(t)$ does not change anymore. 


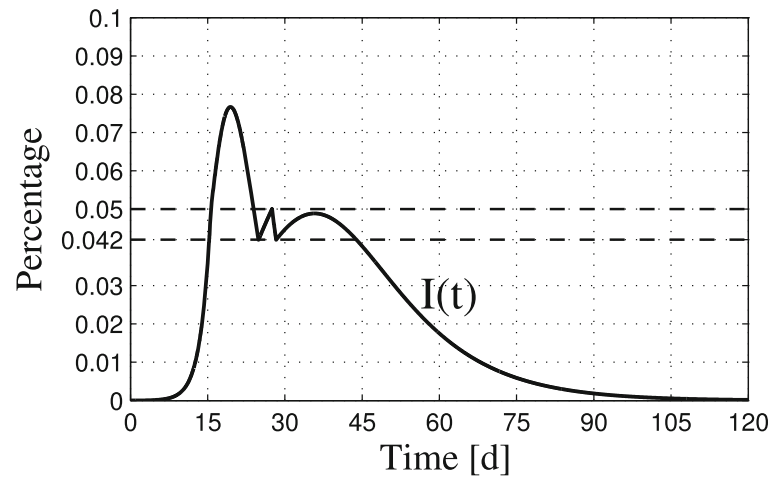

(a)

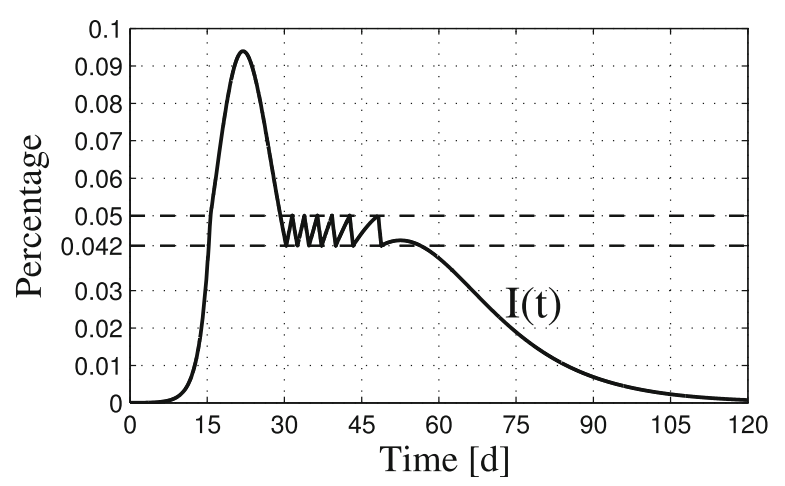

(c)

Fig. 4 System response time for the initial condition $(S(0), I(0), R(0), X(0))=\left(1-10^{-5}, 10^{-5}, 0,0\right)$. We consider $I_{m}=0.05, \alpha=0.7, \beta=0.15$ and: (i) $\kappa=0.2$ and $\kappa_{0}=0.07$,

\section{Concluding remarks}

We provide a dynamic analysis of the model [3] by introducing piecewise smooth vector fields in it. An intermittent protocol is installed according to the number of infected individuals and taking into account the capacity of the health system. As a result, some interesting bifurcations were reported. By answering Question 1 , given a maximum number of infected, we determine the minimum effort of quarantine or both containment and quarantine needed for not collapsing the health system above this number. By answering Question 2, given an effort of containment and isolation, we determine the threshold number of infected individuals above which these measures must be implemented to not collapse the health system.

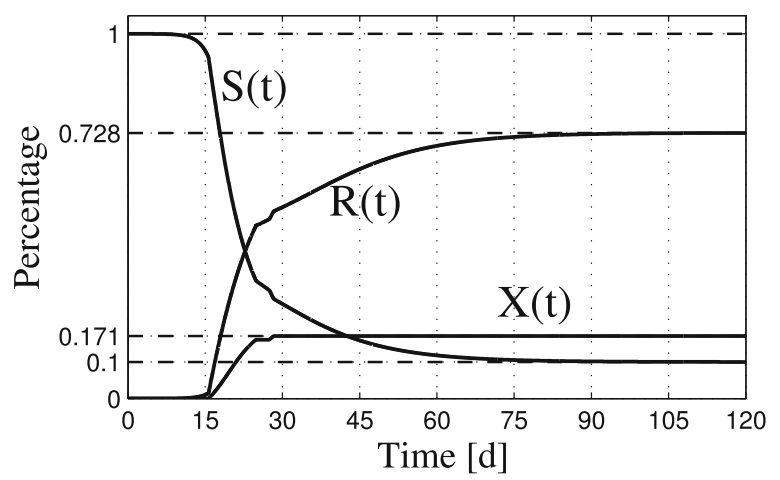

(b)

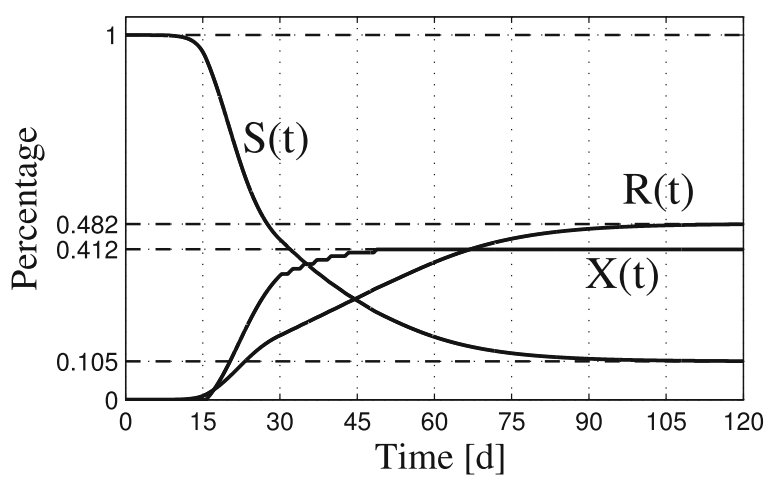

(d)

see $\mathbf{a}-\mathbf{b}$; (ii) $\kappa=0.315$ and $\kappa_{0}=0$, see $\mathbf{c}-\mathbf{d}$. Hysteresis control parameter: $\delta=0.008$

Acknowledgements T. Carvalho is partially supported by grants \#2019/10269-3 and \#2019/10450-0, São Paulo Research Foundation (FAPESP), Conselho Nacional de Desenvolvimento Científico e Tecnológico (CNPq) grant 304809/2017-9. D. J. Tonon is supported by PROCAD/CAPES grant 88881.0 68462/2014-01 and PRONEX/FAPEG grant 2017/10267000508, and D. S. Rodrigues and R. Cristiano thanks PNPD/CAPES.

Data availability Data sharing not applicable to this article as no datasets were generated or analysed during the current study.

\section{Declarations}

Conflict of interest The authors declare that they have no conflict of interest.

\section{References}

1. World Heath Organization. COVID-19 Timeline. Available at: https://www.who.int/emergencies/diseases/ 
novel-coronavirus-2019/interactive-timeline, Accessed: 20/08/2020

2. Randolph, Haley E., Barreiro, Luis B.: Herd immunity: Understanding COVID-19. Immunity 52(5), 737-741 (2020)

3. Benjamin MF, Brockmann D (2020) Effective containment explains subexponential growth in recent confirmed COVID-19 cases in China. Science 368(6492), 742-746

4. Matt J. Keeling and Pejman Rohani. Modeling Infectious Diseases in Humans and Animals. Princeton University Press, 1 edition, 2007

5. James, Lyndon P., Salomon, Joshua A., Buckee, Caroline O., Menzies, Nicolas A.: The use and misuse of mathematical modeling for infectious disease policymaking: Lessons for the COVID-19 pandemic. Medical Decision Making 41(4), 379-385 (2021)

6. Perra, Nicola: Non-pharmaceutical interventions during the COVID-19 pandemic: A review. Physics Reports 913, 1-52 (2021)

7. Estrada, Ernesto: COVID-19 and SARS-CoV-2: modeling the present, looking at the future. Physics Reports 869, 1-51 (2020)
8. Nakamoto I, Zhang J (2021) Modeling the underestimation of COVID-19 infection. Results Phys. 25, 104271

9. Filippov, A. F.: Differential Equations with Discontinuous Righthand Sides, volume 18 of Mathematics and its Applications. Springer Netherlands, first edition, 1988

10. Kuznetsov, Yu.A., Rinaldi, S., Gragnani, A.: One-parameter bifurcations in planar Filippov systems. International Journal of Bifurcation and Chaos 13(8), 2157-2188 (2003)

11. Jacquemard, A., Teixeira, M.A., Tonon, D.J.: Stability conditions in piecewise smooth dynamical systems at a twofold singularity. Journal of Dynamical and Control Systems 19(1), 47-67 (2013)

Publisher's Note Springer Nature remains neutral with regard to jurisdictional claims in published maps and institutional affiliations. 